\title{
El delito en el barrio Virgen de Guadalupe de la ciudad de Formosa, Argentina. ¿Inseguridad real o estigmatización residencial?
}

\author{
The crime in the Virgen de Guadalupe neighborhood \\ of the city of Formosa, Argentina. Real insecurity or \\ residential stigmatization?
}

\author{
Ricardo Omar Conte ${ }^{1}$ \\ Patricia Gabriela Pastor ${ }^{2}$ \\ Matías Sebastián Martínez ${ }^{3}$
}

Fecha de recibido: 12 de julio de 2021

Fecha de aceptado: 21 de septiembre de 2021

\section{Resumen}

El barrio Virgen de Guadalupe es uno de los conglomerados barriales más populosos de la ciudad de Formosa. Es un complejo habitacional de viviendas colectivas construidas mediante el Fondo Nacional de Vivienda (FONAVI) a fines de la década de los setenta, durante la última dictadura militar.

Doctoren Geografía. Profesor Titular Ordinario de la carrera de Geografía, Facultad de Humanidades, Universidad Nacional de Formosa (UNAF). Investigador Categoría II, Secretaría General de Ciencia y Tecnología (SECYT-UNAF), Argentina, correo electrónico: ricardoomarconte@gmail.com

Licenciada en Geografía, doctorado en Ciencia Política y Mágister en Dirección y Gestión Pública Local. Docente de la carrera de Geografía de la Facultad de Humanidades, UNAF-Asociación de Geógrafos de Formosa (GEOFOR), correo electrónico: patriciagabrielapastor@hotmail.com

Licenciado en Geografía. Docente de la Licenciatura en Turismo de la Facultad de la Producción y Medio Ambiente. UNAF-Universidad Nacional de Formosa-Instituto Universitario de FormosaGEOFOR, correo electrónico: mmatiassebastian@gmail.com 
En el imaginario colectivo de los vecinos capitalinos se manifiesta como uno de los sectores urbanos más peligrosos e inseguros de la ciudad de Formosa, solo superado en magnitud de percepción de inseguridad por el barrio Obrero.

El principal objetivo de este artículo es analizar las relaciones existentes entre la ocurrencia de los delitos y las percepciones de inseguridad en el barrio Virgen de Guadalupe.

Metodológicamente, para realizar este trabajo se ha utilizado por un lado una muestra de la población formoseña de 651 individuos relevados mediante encuestas de percepción de inseguridad realizadas a los vecinos, y por otro lado datos de ocurrencia de delitos en la ciudad de Formosa durante el año 2016, suministrados por la autoridad policial.

El principal hallazgo obtenido ha sido la verificación de una situación de estigmatización residencial, producto de la influencia de los medios de comunicación y de las redes sociales.

Concluimos que la situación de estigmatización residencial que sufre el barrio, no se condice con los niveles reales de ocurrencia del delito en dicho barrio, y que la percepción topofóbica del mismo proviene esencialmente de la "mala fama" que arrastra hace un tiempo, y que se traduce en problemas de segregación residencial para sus habitantes.

Palabras clave: estigmatización residencial, percepción, inseguridad, delito, barrio Virgen de Guadalupe.

\section{Abstract}

The Virgen de Guadalupe neighborhood is one of the most populous neighborhood conglomerates of Formosa city. It is a residential complex of collective houses built through the National Housing Fund (FONAVI) in the late 70 s, during the last military dictatorship.

In the collective imagination of the residents of the capital, it appears as one of the most dangerous and insecure urban sectors of the city of Formosa, only surpassed in magnitude of perception of insecurity by the Obrero neighborhood.

The main objective of this article is to analyze the relationships between the occurrence of crimes and the perception of insecurity in the Virgen de Guadalupe neighborhood.

Methodologically, to carry out this work, on the one hand, a sample of the Formosa population of 651 individuals surveyed through surveys of perception of insecurity carried out to the neighbors has been used, and on the other hand, data on the occurrence of crimes in the city of Formosa during the year 2016, supplied by the police authority. 
The main finding obtained has been the verification of a situation of residential stigmatization, a product of the influence of the media and social networks.

We conclude that the situation of residential stigmatization suffered by the neighborhood is not consistent with the real levels of crime in said neighborhood, and that the topophobic perception of it comes essentially from the "bad reputation" that has been dragging on for some time, and that it translates into residential segregation problems for its inhabitants.

Key words: residential stigmatization, perception, insecurity, crime, Virgen de Guadalupe neighborhood.

\section{Introducción}

El barrio Virgen de Guadalupe es uno de los barrios colectivos de viviendas unifamiliares más populosos de la ciudad de Formosa, Argentina. Asimismo, es uno de los sectores residenciales planificados más antiguos de la capital formoseña. El área urbana consolidada de la ciudad tiene 6100 hectáreas de superficie, una densidad de población de 36 hab/ha; mientras que el barrio Guadalupe tiene una superficie de 20 ha y una densidad de $243 \mathrm{hab} / \mathrm{ha}$.

Construido a fines de la década de los setenta en pleno proceso militar en Argentina, posee características edilicias similares al famoso barrio Ejército de los Andes -más conocido como Fuerte Apache-localizado en el conurbano bonaerense. Su distribución en torres (con varios departamentos cada una de ellas) y la disposición simétrica de las mismas entre intrincados pasillos, le otorgan una fisonomía única desde el punto de vista morfológico dentro de la ciudad capital. En los últimos años y en concordancia con lo que sucede en las grandes ciudades del resto del país, el delito ha crecido en la ciudad de Formosa, lo que puede percibirse en la ciudadanía.

Este artículo va a analizar si esta percepción de inseguridad de los vecinos formoseños se corresponde con los índices reales de ocurrencia de delitos en el barrio estudiado, o si éste experimenta una situación de estigmatización residencial, producto de la influencia de los medios de comunicación y de las redes sociales. Para ello se va a tratar la temática del delito con dos puntos de vista: el subjetivo, estudiado desde la percepción de la inseguridad, y el objetivo, analizado desde la ocurrencia del delito.

Es así, que el principal objetivo en este trabajo es analizar las relaciones existentes entre la ocurrencia de los delitos y las percepciones de inseguridad en el barrio Virgen de Guadalupe de la ciudad de Formosa durante el año 2016, considerando como hipótesis de trabajo que este sector residencial se 
encuentra bajo una mirada estigmatizante debido a la influencia negativa de los medios de comunicación y de las redes sociales.

\section{Materiales y métodos}

Para este estudio se han utilizado por un lado, los datos obtenidos de las encuestas de percepción de inseguridad recolectadas durante la ejecución de investigaciones referidas a los tipos de delitos en la capital formoseña acreditado ante la Secretaría General de Ciencia y Tecnología (SECYT) de la Universidad Nacional de Formosa (UNAF) con el código 54H/129 (del cual se desprende este trabajo), y por otro lado, los datos de delito suministrados por el Comando Radioeléctrico de la Policía de la provincia de Formosa correspondientes al año 2016.

En el caso de las encuestas de percepción de inseguridad, dentro de las estrategias dirigidas al diseño del modelo, se han considerado tres tipos de muestreo, los cuales fueron aplicados de forma combinada y que son los siguientes:

- Muestreo aleatorio simple (MAS): de acuerdo a este método todos los individuos o elementos que integran la población o universo tienen la misma probabilidad de ser elegidos para formar parte del modelo. Esta técnica permite calcular el tamaño de la muestra, lo cual se traduce en el número de habitantes y de encuestas que debemos considerar y confeccionar. Se basa en la aplicación y el desarrollo de la siguiente fórmula:

$$
n=\frac{Z^{2} p q N}{N E^{2}+Z^{2} p q}
$$

Para realizar el estudio que comprende a una población de 143850 habitantes, estimando un nivel de confianza del $99 \%$ y un margen de error del $2 \%$, la muestra debe estar compuesta por 610 individuos. El equipo de investigación recolectó unas 651 encuestas.

- Muestreo por cuota: normalmente esta segmentación se hace empleando alguna variable sociodemográfica como sexo, edad, o clase socioeconómica. De esta manera es posible extrapolar, desde el total, el valor proporcional que se le asignará a cada categoría o variable que compone la muestra. En el caso de la segmentación realizada para este trabajo se tomaron las variables edad y sexo, como puede verse en la Tabla 1.

- Muestreo intencional o por juicio: mediante esta técnica de muestreo no probabilístico hemos definido la distribución de la muestra en términos espaciales, es decir por barrios de la ciudad de Formosa.

- Muestreo intencional o por juicio: mediante esta técnica de muestreo no probabilístico hemos definido la distribución de la muestra en términos espaciales, es decir por barrios de la ciudad de Formosa. 
Tabla 1. Composición de la población (edades

quinquenales y sexo), ciudad de Formosa, 2010

\begin{tabular}{|c|c|c|c|}
\hline \multirow[b]{2}{*}{ Municipio } & \multirow{2}{*}{$\begin{array}{c}\text { Edades } \\
\text { quinquenales }\end{array}$} & \multicolumn{2}{|c|}{ Sexo } \\
\hline & & Varón & Mujer \\
\hline \multirow{21}{*}{ FORMOSA } & $0-4$ & 9818 & 9315 \\
\hline & $5-9$ & 10572 & 10120 \\
\hline & $10-14$ & 11885 & 11794 \\
\hline & 15-19 & 12392 & 12638 \\
\hline & $20-24$ & 9263 & 10070 \\
\hline & $25-29$ & 7999 & 8979 \\
\hline & $30-34$ & 7714 & 8779 \\
\hline & $35-39$ & 6651 & 7684 \\
\hline & $40-44$ & 5928 & 6959 \\
\hline & $45-49$ & 5651 & 6341 \\
\hline & $50-54$ & 4982 & 5890 \\
\hline & $55-59$ & 4293 & 4804 \\
\hline & $60-64$ & 3161 & 3672 \\
\hline & $65=69$ & 2388 & 2828 \\
\hline & $70-74$ & 1741 & 2280 \\
\hline & $75-79$ & 1175 & 1616 \\
\hline & $80-84$ & 618 & 1060 \\
\hline & $85-89$ & 281 & 498 \\
\hline & $90-94$ & 110 & 177 \\
\hline & 95 y más & 26 & 66 \\
\hline & Total & 106648 & 115570 \\
\hline
\end{tabular}

Fuente: elaboración propia en base al Censo Nacional de Población y Vivienda 2010. Provincia de Formosa. Instituto Nacional de Estadística y Censos, http://www.indec.gov.ar/

Según la Dirección de Catastro Municipal, la ciudad de Formosa contabilizó en el 2016 un total de 110 barrios, los cuales constituyen las unidades espaciales de aplicación de los instrumentos de recolección de datos, es decir, las encuestas de percepción de inseguridad. La ponderación del número de encuestas por barrio se realizó en forma proporcional a la cantidad aproximada de habitantes de cada barrio.

Por otra parte, los datos referidos a la cantidad de delitos por barrios ocurridos en la ciudad de Formosa fueron suministrados por el Comando Radioeléctrico de la Policía de la Provincia de Formosa. Este organismo registró en el año 2016 la friolera de 5376 delitos en todo concepto.

\section{Desarrallo}

\section{La inseguridad y su percepción ciudadana}

La "inseguridad" es una percepción, es una imagen mental que construye una persona y su grupo, en este caso, sobre la posibilidad de ser víctima de un 
delito en un lugar determinado, es una apreciación subjetiva de un "espacio" que emerge del colectivo imaginario de las personas, el cual es construido en parte, por las experiencias, como también por la información a la que acceden las personas a partir de su red de relaciones, como por diferentes medios de comunicación. Es una apreciación subjetiva de un lugar mediante el colectivo imaginario de las personas. Es claramente una manifestación topofóbica respecto a un espacio determinado.

Este tema genera un amplio debate en la agenda pública y de los medios de comunicación en el país y en el mundo, pasando a ser una de las preocupaciones prioritarias de la población según varios estudios, principalmente la de grandes urbes, aunque no escapan a esa preocupación, ciudades intermedias como Formosa.

Según Fraile (2007) la relación entre la organización territorial y las sensaciones y actitudes que en ese territorio se manifiestan de diferentes maneras, siendo la imagen del entorno -analizada por Lynch y cuyas manifestaciones son los mapas mentales- la más conocida. La percepción del entorno condiciona la actividad cotidiana de los individuos y su vinculación con el medio, pudiéndose generar repercusiones negativas respecto la sensación de seguridad experimentada en un determinado entorno, "influyendo en el uso que de él se hace, generando así una espiral que acaba estigmatizando algunos sitios o estimulando la frecuentación de otros" (p. 1).

Las zonas más densamente urbanizadas del país vienen sobrellevando problemas estructurales que son de tipo: económicos, políticos y sociales, y tienen dispares grados de impacto en la población (desocupación, corrupción, pobreza, limitaciones a la educación formal, entre los más importantes). Estos problemas profundizan la descomposición de la sociedad y sus valores comunitarios y constituyen una puerta abierta a las diferentes formas delictivas. La inseguridad posee diversas dimensiones y facetas y amerita ser analizada tanto objetiva como subjetivamente, en nuestro campo, esos problemas pueden ser abordados espacialmente.

Es por ello que esta problemática ha despertado el interés de la Geografía, enalteciendo a la vez su importancia como ciencia capaz de generar un corpus teórico-metodológico ineludible para un abordaje integral de la inseguridad.

La importancia y necesidad de investigaciones de esta naturaleza, que en el contexto local pretende constituir un punto de inflexión -ya que se registran pocos antecedentes relacionados a esta problemática- radica en la posibilidad de generar resultados que sean útiles tanto a nivel cognitivo, asociado a la Geografía como ciencia social y aplicada como también en el orden socio-institucional, es decir, como insumo para contribuir a la toma de decisiones a nivel territorial por parte de las diversas instituciones públicas que posean competencia. 
A fines del siglo pasado los estudios acerca de la inseguridad comienzan a integrar líneas de estudio referidas al miedo al delito, la victimización de las personas, la estigmatización residencial, entre otras.

Van Soomeren (2007) considera que las grandes áreas urbanas han aumentado sus tasas delictivas y sus problemas de seguridad, lo que trae aparejado un aumento del sentimiento de inseguridad, traducido en la reducción de la calidad de vida de los residentes urbanos.

Kessler (2009) afirma que el delito produce temor en las personas, siendo que la criminalidad puede encontrarse en todos lados, en todas las sociedades, globalizándose de tal manera que la seguridad es considerada por la Organización de las Naciones Unidas (ONU) como un indicador de calidad de vida.

La inseguridad tiene que ver con hechos delictivos, como también con los sentimientos tales como la ira, el miedo, el condicionamiento de las libertades, la indignación y la expectativa de ser victimizado, siendo que para ello se llevan a cabo una serie de acciones individuales y colectivas para enfrentarse a las amenazas percibidas (Bergman y Kessler, 2008).

Moreno (2012) explica que la percepción de inseguridad es una sensación de amenaza azarosa, siendo que los individuos que la experimentan consideran que alguna vez pueden ser victimizados, lo que produce situaciones de aislamiento, fobia, pánico, alterando de esa manera la integridad, libertad y seguridad de las personas, lo que se ve reflejado en el acrecentamiento de la pérdida de cohesión social.

Las ciudades, son espacios diversos, con diferentes usos en cada sector, es por ello que no son consideradas como peligrosas por sus habitantes en toda su extensión, sino más bien existen determinados lugares o sectores percibidos como inseguros, manifestándose en estos lugares los mismos sentimientos topofóbicos (Segura, 2009).

Según Mape Guzmán et. al (2017), y Capron (2009), los espacios públicos en donde ocurrieron frecuentes hechos delictivos son configurados como lugares o territorios del miedo en el imaginario popular, lo que genera conductas ciudadanas de rechazo hacia los mismos. Estos sectores, se constituyen como fragmentos espaciales dentro de una ciudad y están asociados a sensaciones topofóbicas de diversa jerarquía (Lindón, 2007).

Barinas Salcedo (2014) considera a estos territorios del miedo, como una experiencia espacial de percepción sensorial, lo que genera que este imaginario popular influya tanto en forma individual como colectiva en las formas y prácticas sociales cuya finalidad es evitar el miedo al delito.

Conte (2017) asegura que la victimización y la percepción de la inseguridad - a pesar de ser interdependientes - no siempre transitan caminos paralelos, ya que muchas veces es disímil la cantidad de delitos cometidos y la sensación 
de inseguridad percibida, siendo que la última puede verse alterada por factores intrínsecos y extrínsecos que modifican la noción de riesgo delictivo.

Para Vera et al. (2017) el temor a ser victimizado y la sensación de inseguridad se relacionan tanto con los índices delictivos como con la información con que cuentan los habitantes mediante numerosos canales comunicativos del mundo actual: las nuevas tecnologías, los medios de comunicación tradicionales y modernos y la interacción social de persona a persona, como a través de diferentes medios.

\section{La estigmatización residencial como indicador de segregación barrial}

La estigmatización residencial puede traer aparejada como consecuencia la segregación residencial, que es el estadio social más indeseado por los habitantes de un barrio. Segura (2009) afirma que "la segregación residencial no es un fenómeno reciente; por el contrario, es en sus distintas modalidades de segregación residencial socioeconómica, racial o étnica, un rasgo constitutivo de la ciudad capitalista" (p. 1).

Elorza (2019) conceptualiza segregación residencial "como un proceso de desigual distribución espacial de bienes y servicios, como también las construcciones de sentidos (representaciones, descalificaciones, estigmatizaciones, etcétera) que se constituyen como fundamentos de ellos" (p. 93).

Entre las diferentes formas de segregación residencial Carman et. al (2013) distingue la segregación acallada (cuyo efecto es la exclusión de sectores populares), la segregación por default (produce efectos sociales negativos por falta de asistencia estatal), la segregación indolente (materializada en procesos de autosegregación de las clases altas en barrios cerrados suburbanos) y segregación agravada (refiere a procesos y situaciones de confinamiento socio-espacial interiorizados por la hostilidad que supone la segregación social).

Pyszczek (2012) considera en una primera instancia que el sentimiento de inseguridad conlleva a la estigmatización, siendo esta es un proceso de percepción producto de experiencias directas e indirectas de las personas con el medio urbano. En una segunda instancia este autor asevera que las percepciones son afirmadas asociativamente con las experiencias de vida. Aquí se incluye la conformación de estructuras cognitivas que ordenan y dan significado a las vivencias del espacio. En un orden general conforman estructuras o representaciones que se extienden y perpetúan espacial y temporalmente, siendo las mismas tanto de carácter individual como colectivo. 
En los barrios, la estigmatización genera una relación ambivalente de los habitantes con su propio vecindario, traducida en una actitud de rechazo y de defensa del barrio a la vez. El estigma es una experiencia individual y colectiva muy difícil de sobrellevar. Wacquant et. al (2014) explican que el concepto de estigma territorial conlleva a una relación entre pobreza, barrios marginales, delincuencia callejera y subordinación étnica que incluyen las minorías (grupos inmigrantes, clases sociales bajas).

Segura (2009) afirma que la estigmatización posee efectos negativos tanto hacia afuera como hacia dentro de un barrio. Hacia afuera, la misma produce un proceso de naturalización de la desigualdad (deslegitimación de reclamos, dificultad de acceso a los servicios públicos, como d salud y transporte) y por último desincentiva la circulación del resto de los habitantes de la ciudad por tales espacios barriales, estimulando una segregación residencial periférica que incluye asentamientos marginales y sus residentes. Hacia adentro la estigmatización barrial potencia la conflictividad interna, estimula la evitación mutua y la desconfianza interpersonal. Las esquinas y baldíos de los barrios son ocupados por jóvenes que se apropian de ellos, generando temor entre los vecinos y transeúntes.

En definitiva, el autor explica la estigmatización territorial como una dimensión simbólica de la desigualdad socio-espacial. Por un lado, tiende a naturalizar una geografía urbana dispar, reproduciendo las distancias y la segregación con el resto de los habitantes de la ciudad, potenciado esto por obstáculos económicos (financieros) y geográficos (circulatorios), dificultando las posibilidades de articulación entre residentes que enfrentan problemas comunes. Lamentablemente, cuando un barrio es estigmatizado, su población, que solía enfrentar obstáculos para la gestión de sus problemas estructurales, empeora su situación y le es muy difícil sacarse ese estigma de encima.

El "etiquetamiento" social de un barrio alcanza tanto a los delincuentes como al resto de los vecinos honrados, ya que como expresan Bergman et al. (2008) la sensación de inseguridad se refleja en los altos índices delictivos, pero cuando estos índices disminuyen el sentimiento topofóbico hacia ese barrio continúa perdurando temporalmente.

\section{Resultados}

\section{Antecedentes recientes respecto a la temática de estudio}

Existen numerosos trabajos referidos a la percepción de la inseguridad y a la estigmatización residencial en la Argentina y en América Latina. Conte (2016) llevó a cabo un estudio de percepción ciudadana sobre la inseguridad y estigmatización en el barrio Obrero de la ciudad de Formosa. 
Martínez (2020) trae a colación su tesina de licenciatura sobre percepción de inseguridad y estigmatización residencial referida al barrio en estudio, Virgen de Guadalupe, que sirve de sustento a este artículo.

Kessler (2012) analizó la situación de estigmatización residencial de un complejo habitacional planificado del conurbano bonaerense, el barrio Ejército de los Andes, en la localidad de Caseros.

También Pyszczek (2012) llevó a cabo un trabajo referido a los espacios subjetivos del miedo, analizando el caso de estigmatización residencial de dos barrios de la ciudad de Resistencia, el barrio Guiraldes (barrio de morfología similar a Fuerte Apache) y Villa Prosperidad, uno de los asentamientos de emergencia más antiguos de la capital chaqueña.

Flores Gutiérrez (2015) escribió una tesis de maestría donde recabó datos de percepción de inseguridad y miedo al delito, en el marco de la elaboración de diagnósticos de inseguridad. La misma tuvo como objeto de estudio la delegación Coyoacán y la colonia Santo Domingo, en la Ciudad México.

Fuentealba Carrasco et al. (2016) analizaron para la ciudad de Concepción, Chile, el fenómeno de la sensación de inseguridad y su relación con otras variables (probabilidades de victimización y preocupación por ser víctima) en diferentes estratos sociales y económicos de esa ciudad chilena.

Mape Guzmán et al. (2017) estudiaron topofobias y los imaginarios del miedo en Fontibón, Colombia.

Por otra parte, entre los antecedentes de estudios referidos a segregación residencial, podemos mencionar los aportes de Elorza (2019), que realizó una comparación dentro de la ciudad de Córdoba entre un barrio de viviendas sociales y un asentamiento de emergencia surgido de una usurpación de tierras.

También Tecco y Fernández (2008) llevaron a cabo también en la ciudad de Córdoba, una investigación sobre segregación residencial cuyo eje estuvo enfocado en el agrupamiento espacial de hogares con características semejantes en sus rasgos económicos, sociales y educativos. Los autores argumentan que la fragmentación social y espacial estimulan diferencias sociales en el colectivo urbano.

Segura (2012) realizó un trabajo sobre segregación residencial socioeconómica en un barrio de la ciudad de La Plata relacionando clases sociales y espacios urbanos en la periferia platense.

\section{El área de estudio}

Conte (2000) describe a la ciudad de Formosa como una ciudad ribereña enclavada a orillas del río Paraguay, asentada sobre una llanura aluvial de suelos limo-arcillosos que constituye parte de una sabana-parque en una región de clima cálido subtropical. 
El sitio urbano está rodeado hacia el sur por riachos, esteros y lagunas que frenan la expansión urbana (al igual que en el este por donde discurre el río Paraguay) por lo que la misma se extiende hacia el norte y el oeste.

En la Figura 1, se puede ver la localización geográfica del barrio Virgen de Guadalupe dentro de la ciudad de Formosa y las características fisiográficas del sitio de la capital formoseña.

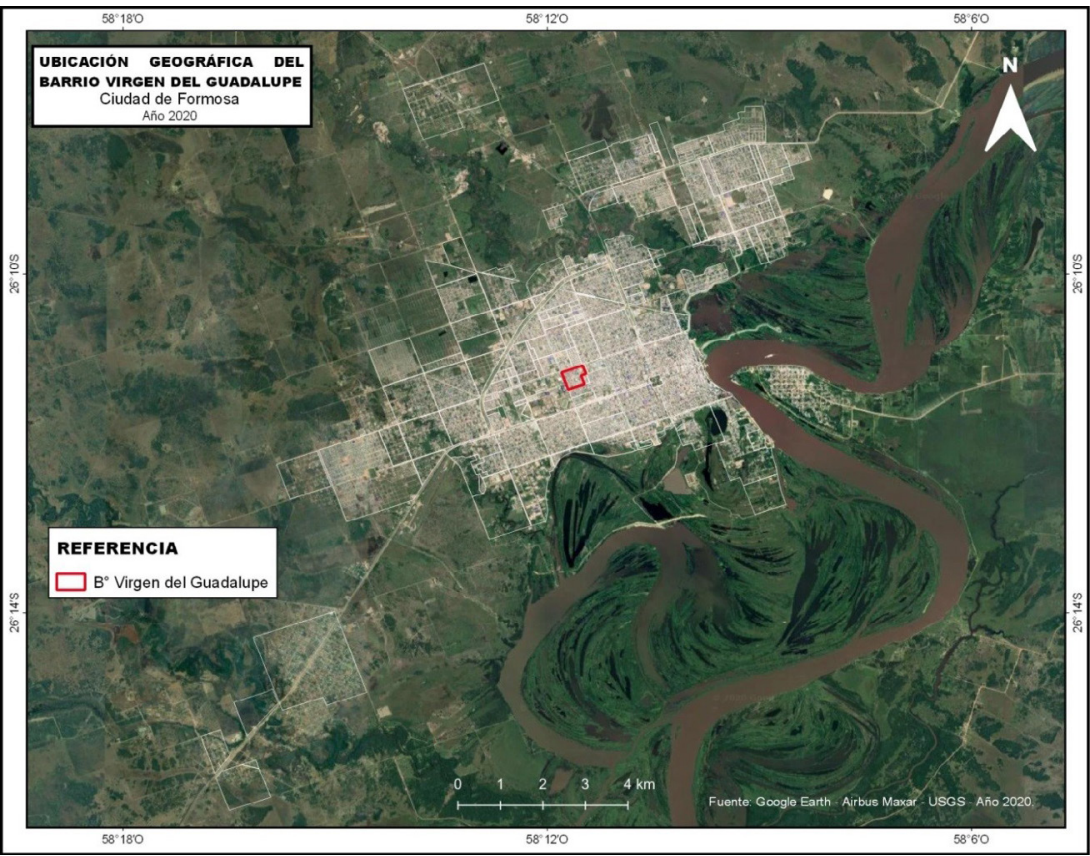

Figura 1. Localización espacial del barrio Virgen de Guadalupe en la ciudad de Formosa Fuente: Imagen satelital Google Earth, 2020.

Con respecto al barrio Virgen de Guadalupe, el objeto de estudio, Martínez (2020) explica que el mismo se caracteriza por ser un barrio planificado de viviendas colectivas del Fondo Nacional de Vivienda (FONAVI) construido a fines de la década de los años setenta por la empresa Seidman y Bonder, y posee departamentos dispuestos en torres modulares similares al barrio Ejército de los Andes (conocido popularmente como Fuerte Apache) en el conurbano bonaerense. El complejo habitacional cuenta con unas 1400 unidades habitacionales, donde habitan unas 8000 personas aproximadamente.

El barrio se encuentra bordeado por las avenidas Néstor Kirchner, Antártida Argentina, la calle Maipú, la calle Armada Nacional, la calle Juan José Silva y la calle Policía de Territorios Nacionales, ubicación que hoy podría considerarse 
céntrica, con un gran movimiento de personas y flujo vehicular a diario y a toda hora.

En la Figura 2 se puede observar que el barrio se encuentra próximo a servicios y equipamientos que generan alto tráfico. Un ejemplo es el Hospital de Alta complejidad Juan Domingo Perón, el Mercado Frutihortícola, el polideportivo Estadio Cincuentenario, el Centro Polivalente de Artes, la EPES núm. 51, además de contar internamente con una escuela primaria: EPES núm. 398 y una escuela Secundaria EPES núm. 27.

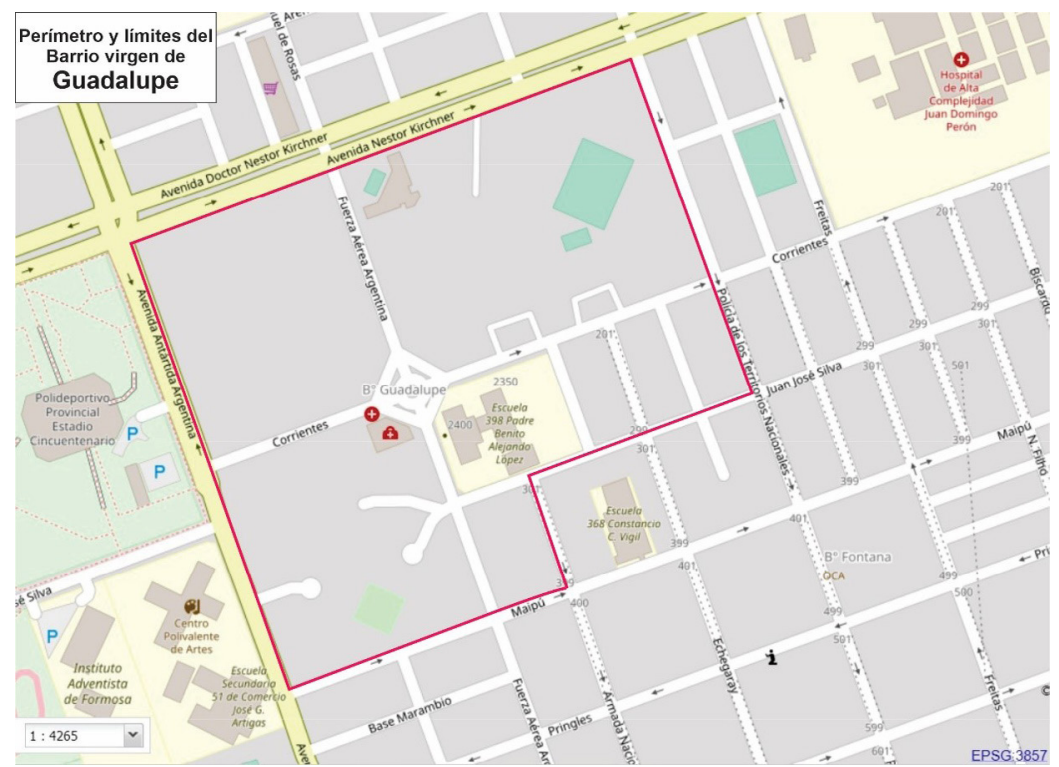

Figura 2. Perímetro y delimitación del barrio Virgen de Guadalupe.

Fuente: Infraestructura de Datos Espaciales de Formosa, IDEF, 2020.

Este barrio tiene una estructura de propiedad horizontal, edificios en una planta baja y dos altas, un sistema de núcleo de escaleras para acceder de circulación vertical. Construido en base a una horma que poco se ajusta a la idiosincrasia de la ciudad de Formosa, "El Guada", como se lo conoce popularmente, consta de torres amontonadas con pasillos y escaleras oscuras unidas por puentes - que a la vez son habitaciones de alguien - que flotan sobre las calles más transitadas (Figura 3a, Figura 3b, Figura 3c y Figura 3d; además de Figura 4a y Figura 4b). Es un verdadero laberinto construido en una ciudad donde sobran los espacios, solo para dimensionar, el espacio urbano consolidado tiene $61 \mathrm{~km}^{2}$ y el ejido de la ciudad cuenta con $356 \mathrm{~km}^{2}$. 


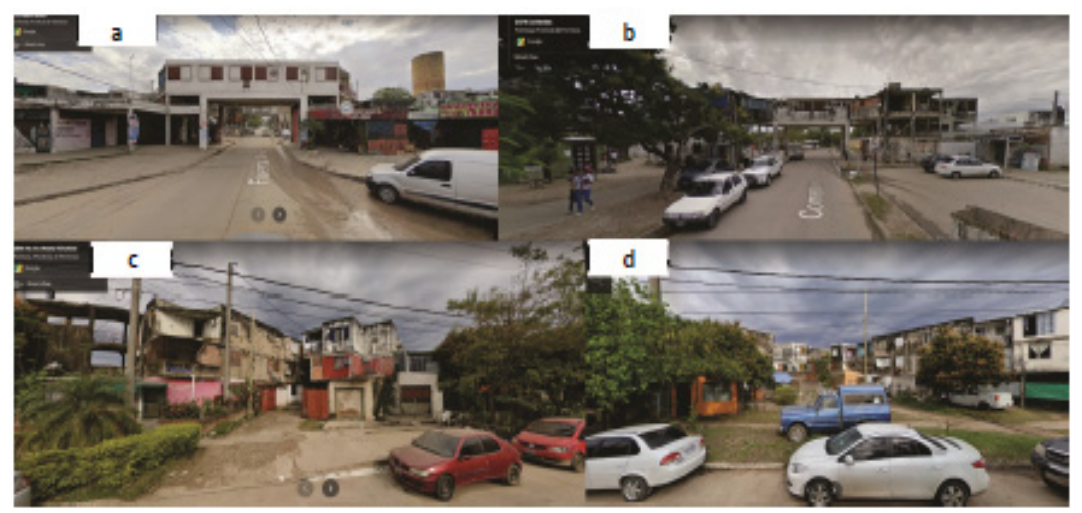

Figura 3. Fachadas de los bloques de departamentos y viviendas "puente" del barrio Virgen de Guadalupe. Ciudad de Formosa.

Fuente: Google Street View (julio, 2019).
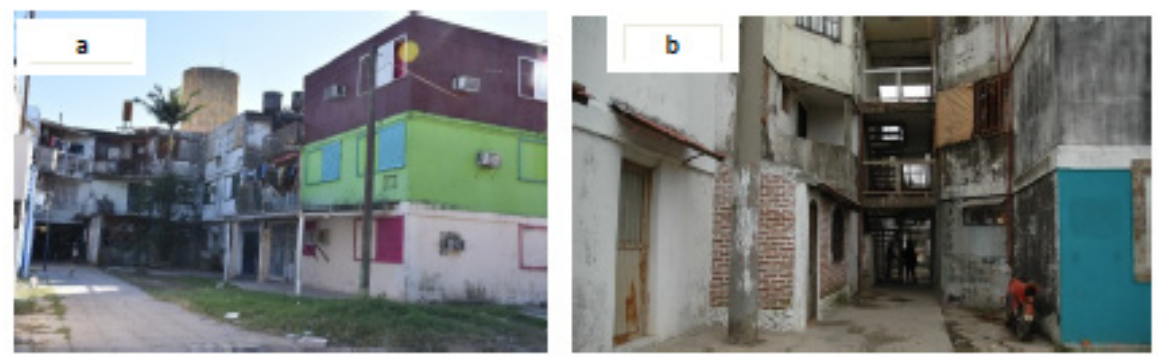

Figura 4. Viviendas y pasillos del barrio Virgen de Guadalupe. Ciudad de Formosa. Fuente: Diario Noticias Formosa.

Como todo barrio de viviendas sociales en la provincia de Formosa es administrado por el Instituto Provincial de la Vivienda (IPV), organismo gubernamental que está llevando a cabo un reordenamiento territorial, que consiste en la desocupación y embellecimiento de los espacios verdes barriales $\mathrm{y}$ en el ordenamiento catastral y dominial de las viviendas oportunamente adjudicadas.

El barrio Virgen de Guadalupe tiene problemas estructurales como el mal funcionamiento de los desagües cloacales; culturales, como el tratamiento de la basura, y otros que - con las políticas correspondientes- podrían mejorar sustancialmente, como ser la seguridad, la circulación interna y el tránsito vehicular. La recuperación de espacios comunitarios, recreativos, estacionamientos y otros mediante un plan de embellecimiento de los mismos, está cambiando la fisonomía de este sector residencial, convirtiéndolo en un lugar mucho más agradable para vivir. 


\section{La percepción de la inseguridad en el barrio Virgen de Guadalupe}

Para obtener los resultados respecto a la percepción de inseguridad en torno al barrio Virgen de Guadalupe se llevó a cabo la aplicación de las encuestas. En una de las preguntas realizadas, la cual pretendía indagar sobre lugares topofóbicos, se les pidió a los vecinos de la ciudad, que mencionen en orden jerárquico descendente de inseguridad los cinco barrios considerados por ellos como más los inseguros de la ciudad de Formosa.

Otra cuestión sobre la que se indagó, fueron las causas que los llevaron a la jerarquizaron los cinco barrios seleccionados. Entre los criterios figuran: en primer lugar, la experiencia personal con hechos de inseguridad en los mencionados barrios, en segundo lugar, los comentarios sobre experiencias de inseguridad relatados por otros residentes y, en tercer lugar, el constante señalamiento a que son sometidos dichos barrios mediante los medios de comunicación gráficos, radiales y televisivos y las redes sociales (la Tabla 2, ofrece un modelo).

Tabla 2. Aspectos/criterios selectivos de inseguridad barrial

\begin{tabular}{llcl}
\hline \multirow{2}{*}{ Nombre del barrio } & \multicolumn{3}{c}{$\begin{array}{c}\text { Aspectos/criterios selectivos } \\
\text { de inseguridad barrial }\end{array}$} \\
\cline { 2 - 4 } & $\mathrm{A}$ & $\mathrm{B}$ & $\mathrm{C}$ \\
\hline 1.- Simón Bolívar & $\mathrm{X}$ & & \\
\hline 2.- Eva Perón & & $\mathrm{X}$ & \\
\hline 3.- Liborsi & & $\mathrm{X}$ & $\mathrm{X}$ \\
\hline 4.- Mariano Moreno & & & $\mathrm{X}$ \\
\hline 5.- Juan Domingo Perón & & & \\
\hline
\end{tabular}

Fuente: Proyecto de investigación SECYT-UNAF 54/H129. Geodimensionando al delito. La ciudad de Formosa y los territorios de la inseguridad. Informe final.

La Tabla 3, refleja la percepción de los vecinos capitalinos respecto a los barrios considerados como más inseguros de la capital formoseña, reflejado en la cantidad de menciones topofóbicas expresadas por los mismos en las 651 encuestas realizadas en toda la ciudad de Formosa.

Ahora bien, considerando la sumatoria de las cinco opciones de percepción de inseguridad barrial que ofrece la encuesta, los barrios Obrero, ubicado al sur, Guadalupe, en el centro de la planta urbana, 20 de Julio y Antenor Gauna -localizados al norte- son considerados los barrios más inseguros de la ciudad. 
Tabla 3. Número de menciones dentro de los cinco barrios más inseguros (3 255 menciones en 651 encuestas)

\begin{tabular}{clcc}
\hline Orden & \multicolumn{1}{c}{ Barrio } & $\begin{array}{c}\text { Número de } \\
\text { menciones }\end{array}$ & $\begin{array}{c}\text { \% sobre } \\
\text { el total }\end{array}$ \\
\hline 1 & Obrero & 235 & 35,6 \\
\hline 2 & Virgen de Guadalupe & 205 & 31,5 \\
\hline 3 & Antenor Gauna & 189 & 29,1 \\
\hline 4 & 20 de Julio & 157 & 24,1 \\
\hline 5 & Simón Bolívar & 147 & 22,4 \\
\hline 6 & Eva Perón & 145 & 22,3 \\
\hline 7 & San José Obrero & 137 & 21,1 \\
\hline 8 & San Agustín & 132 & 20,3 \\
\hline 9 & La Floresta & 126 & 19,3 \\
\hline 10 & El Porvenir & 114 & 17,5 \\
\hline
\end{tabular}

Fuente: elaboración propia con datos del proyecto de investigación SECYT-UNAF 54/ H129. Geodimensionando al delito. La ciudad de Formosa y los territorios de la inseguridad. Informe final.

Se aprecia que más del $31 \%$ de los vecinos perciben al complejo habitacional estudiado (205 percepciones topofóbicas de inseguridad, $2^{\circ}$ lugar en la grilla) como uno de los más inseguros de la capital provincial.

Asimismo, se puede observar en el sector norte una mayor cantidad de barrios considerados inseguros por los vecinos de la ciudad de Formosa y en donde se destacan - además de los antes mencionados- los barrios El Porvenir, Simón Bolívar, Eva Perón, República Argentina, La Floresta y San Agustín, con un grado alto de percepción de inseguridad. En el sector sur, además del barrio Obrero, el barrio San José Obrero presenta también un alto grado de este tipo de topofobia.

Con una sensación menor en lo que respecta a la percepción de inseguridad, se tiene una serie de sectores residenciales dispuestos en forma excéntrica al centro urbano de la ciudad capital, como ser los barrios 12 de Octubre (oeste) Mariano Moreno (sur) San Antonio, Juan Domingo Perón, 7 de Mayo y El Palomar (norte) El resto de los barrios capitalinos, incluyendo el casco céntrico, poseen una sensación de inseguridad de baja a nula. Analizando los criterios de selección de percepción de inseguridad mediante los 205 señalamientos topofóbicos que recibió el barrio Virgen de Guadalupe por parte de los vecinos capitalinos, en más de la mitad (105 señalamientos $-51,2 \%$ ) se reconoció la influencia negativa de los medios de comunicación social - gráficos, escritos o digitales, radiales o televisivos - y de las diversas redes sociales existentes. Se 
destaca que cuando una gran mayoría de los encuestados señala este aspecto o criterio de selección de inseguridad, lo más probable es que estemos ante una situación de estigmatización barrial.

Esta situación se la puede observar en el plano de la Figura 5.

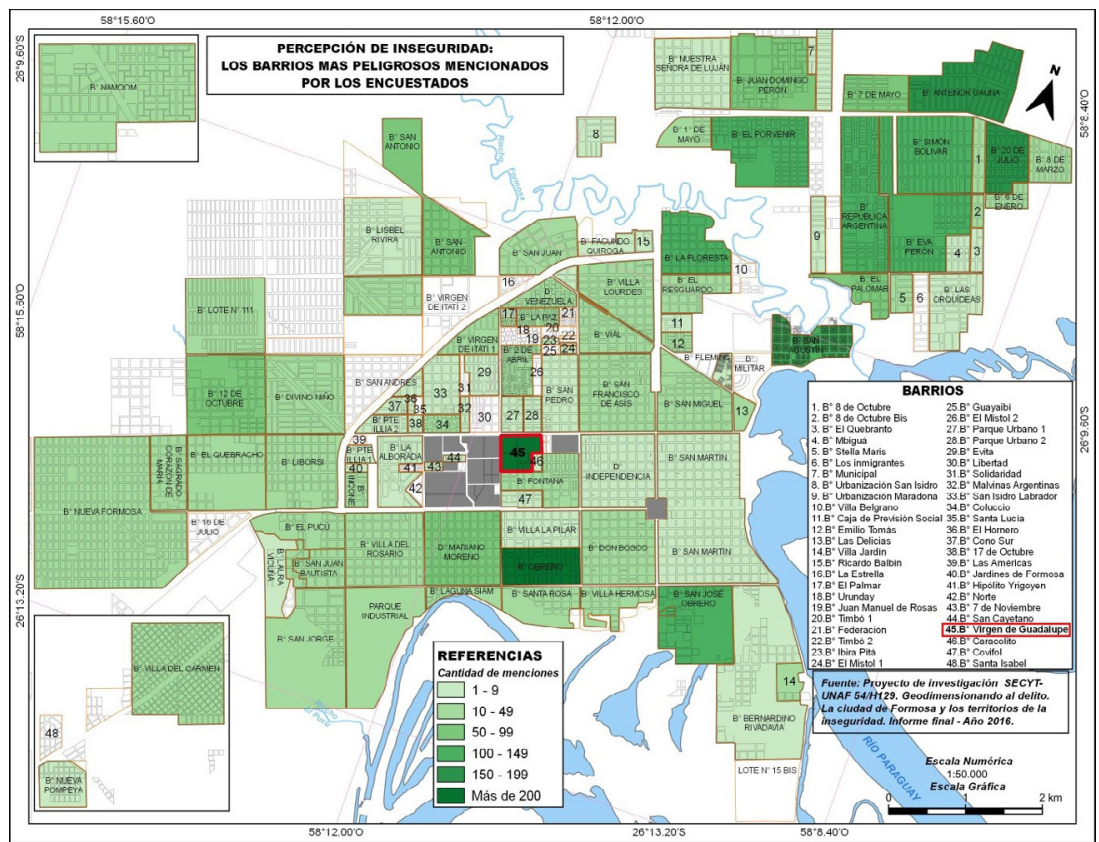

Figura 5. Percepción de inseguridad. Los barrios más inseguros mencionados por los encuestados (3 255 menciones en 651 encuestas).

Fuente: Proyecto de investigación SECYT-UNAF 54/H129. Geodimensionando al delito. La ciudad de Formosa y los territorios de la inseguridad. Informe final.

Una buena parte de los encuestados señalaron negativamente al barrio en estudio por haber escuchado comentarios de terceras personas, (parientes, amigos, conocidos) que a su vez vieron o escucharon comentarios respecto a la inseguridad del mismo (89 señalamientos -43,4\%)

Solo una minoría (11 señalamientos $-5,4 \%$ ) expresó que la consideración de inseguridad de dicho barrio estuvo condicionada a experiencias propias de inseguridad, es decir por haber sufrido algún tipo de delito en carne propia. Esta situación se puede apreciar en la Tabla 4 y en el gráfico de la Figura 6.

Según Urteaga (2012) la estigmatización residencial genera una relación ambivalente de rechazo y defensa respecto del barrio por parte de los vecinos, existiendo asimismo una sensibilidad por parte de estos ante las imágenes 
Tabla 4. Aspectos o criterios de selección y jerarquización respecto a la inseguridad en el barrio Virgen de Guadalupe (número de veces señalado por los encuestados)

\begin{tabular}{lcc}
\hline \multicolumn{1}{c}{ Criterios } & Total & $\%$ \\
\hline $\begin{array}{l}\text { Por experiencia personal de haber sufrido y/o } \\
\text { presenciados hechos de inseguridad en dichos barrios }\end{array}$ & 11 & $5,4 \%$ \\
\hline $\begin{array}{l}\text { Por comentarios realizados al encuestado respecto } \\
\text { a hechos de inseguridad sufridos y/o presenciados } \\
\text { por familiares, amigos y/o terceros en dichos barrios }\end{array}$ & 89 & $43,4 \%$ \\
\hline $\begin{array}{l}\text { Por la frecuente mención de hechos de } \\
\text { inseguridad ocurridos en dichos barrios en } \\
\text { los diferentes medios de comunicación social } \\
\text { gráficos, televisivos y radiales y redes sociales }\end{array}$ & 105 & $51,2 \%$ \\
\hline Total de señalamientos de los encuestados & 205 & $100 \%$ \\
\hline
\end{tabular}

Fuente: elaboración propia con datos del proyecto de investigación SECYT-UNAF 54/ H129. Geodimensionando al delito. La ciudad de Formosa y los territorios de la inseguridad. Informe final.

\section{CRITERIOS DE SELECCIÓN DE PERCEPCIÓN DE INSEGURIDAD. BARRIO VIRGEN DE GUADALUPE}

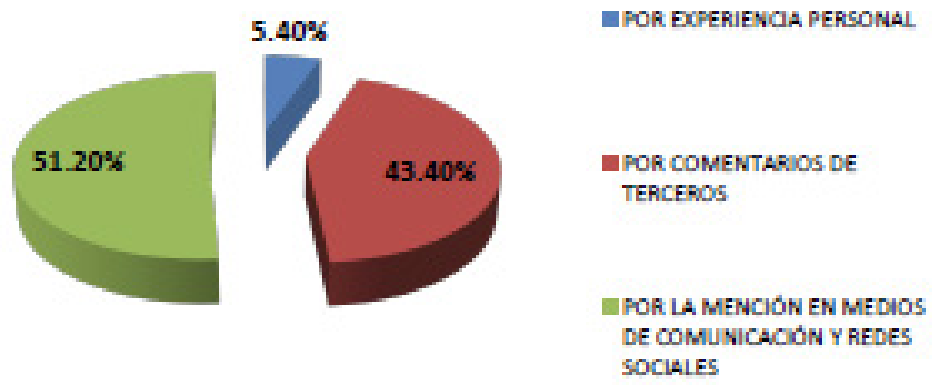

Figura 6. Criterios de selección de percepción de la inseguridad en el barrio Virgen de Guadalupe.

Fuente: elaboración propia con datos del proyecto de investigación SECYTUNAF 54/H129. Geodimensionando al delito. La ciudad de Formosa y los territorios de la inseguridad. Informe final.

negativas provenientes del exterior, provocadas por la reputación negativa, lo que genera una constante preocupación para escapar a la misma.

A modo de ejemplo, puede verse cómo se refleja en la red social Facebook una noticia aparecida en el portal digital formoseño Noticias Formosa respecto a un robo a estudiantes secundarios en los pasillos del barrio Virgen de Guadalupe el 29 de septiembre de 2016: 
Salen de los pasillos del barrio Guadalupe para robar celulares a alumnos de 12 y 13 años del Centro Polivalente de Arte

Hoy a las 10.30 horas de la mañana un grupo de niños, de entre 12 y 13 años, que salían de la clase de gimnasia del Colegio Polivalente de Arte, fueron alcanzados por dos jóvenes que salieron de unos de los pasillos del barrio Guadalupe contó la madre de uno de los niños víctimas del robo de las mochilas y celulares. $L \quad O$ amenazaron con un cuchillo a los niños para robarles las mochilas y los celulares, agregó. Tanta fue la impunidad que se dirigieron caminando de nuevo al pasillo de donde salieron, explicó.

Quiero recalcar que no es la primera vez que pasa esto. Siempre, en avenida Kirchner y Antártida Argentina suelen asaltar a personas que salen a caminar o a chicos que salen del colegio. Por favor, pedimos presencia policial en el lugar a la hora de salida de los chicos del colegio, finalizó. Adelantó que esta tarde vamos a realizar la denuncia y el pedido formal a la sub-comisaría del barrio. (https://www.facebook.com/NoticiasFormosa/posts/denuncian-inseguridad-enel-barrio-guadalupe /634441880062871/)

Comentario de Jessy D. Estudiante. Vive en la ciudad de Formosa El Fuerte Apache de Formosa...

Comentario de Sergio M. Empleado. Vive en la ciudad de Formosa. ¿Barrio? Eso no tiene nada de barrio, más bien villa, antro, aguantadero, nido menos un barrio ese lugar.

Comentario de Andrea G. Estudiante. Vive en la ciudad de Formosa.

Hay que demoler ese lugar. Es tierra de nadie los delincuentes tienen entre 10 y 16 años la Policía los lleva y los padres se van a retirarlo 2 o 3 veces por noche esto es cuestión de los padres. Creo que la ley debería caer sobre ellos para que puedan entender el daño que les están causando a sus hijos y ellos a la sociedad. Mano dura para los padres...

Respuesta de Gabi Z. Estudiante. Vive en el barrio Virgen de Guadalupe.

Es mi barrio, y no siempre los delincuentes que andan por acá son de acá. Y si vamos al caso en todos los barrios te afanan, (roban) si empezamos a nombrar va a ver una lista larga. Bien ignorantes son a la hora de escribir algunos comentarios.

Respuesta de Maru Z. Docente. Vive en el barrio Virgen de Guadalupe.

Por favor que manga (multitud) de gente ignorante la que habla sin saber los delincuentes están en todos lados sea cual sea el barrio no denigren a un lugar donde también vive gente honesta y trabajadora. Capaz que aquellos que hablan tienen a los delincuentes en sus propias casas sin saberlo, ¡cuidado!

Respuesta de Solecita M. Ama de casa. Vive en el barrio Virgen de Guadalupe. Qué saben manga de ignorantes burros, no por culpa de unos cuantos chorros (ladrones) que siempre hay en todos los barrios de Formosa ustedes le meten a todos en la misma bolsa... conozco el barrio y ahi hay gente muy laburadora (trabajadora) también... (Noticias Formosa, 29 de septiembre de 2016). 
Esta conversación llevada a cabo entre usuarios de esa red social pone de manifiesto una de las tantas situaciones de estigmatización residencial que suceden en el barrio Virgen de Guadalupe, lo que puede verificarse en las respuestas de los residentes del barrio, que denotan claramente el enojo y el malestar que les producen los comentarios estigmatizantes ya que, como explica Urteaga, se evidencian los sentimientos y actitudes ambivalentes de "rechazo" y "defensa" del barrio por parte de distintos actores.

\section{La ocurrencia del delito en el barrio Virgen de Guadalupe}

Para llevar a cabo un análisis de la ocurrencia del delito en el barrio Virgen de Guadalupe de la capital formoseña, se han utilizado los datos suministrados por el Comando Radioeléctrico de la Policía de la Provincia de Formosa, cuya información proviene de la línea telefónica 911.

De acuerdo a los datos del año 2016, en la ciudad de Formosa, se cometieron en ese año 5376 delitos, de los cuales unos 975 hechos delictivos $(18,2 \%)$ se llevaron a cabo contra las personas, y unos 4.401 delitos $(81,8 \%)$ contra la propiedad.

La Tabla 5 nos muestra a modo de síntesis los 25 barrios capitalinos que más delitos han sufrido en el período de tiempo analizado.

Tabla 5. Número de hechos delictivos contra la propiedad y contra las personas discriminadas por barrios, 2016 (sobre 5376 delitos)

\begin{tabular}{clcccc}
\hline Orden & Barrios & $\begin{array}{c}\text { Delitos } \\
\text { contra las } \\
\text { personas }\end{array}$ & $\begin{array}{c}\text { Delitos } \\
\text { contra la } \\
\text { propiedad }\end{array}$ & $\begin{array}{c}\text { Total de } \\
\text { delitos }\end{array}$ & $\begin{array}{c}\text { \% de } \\
\text { delitos }\end{array}$ \\
\hline 1 & San Martín & 50 & 475 & 525 & 9,76 \\
\hline 2 & Eva Perón & 88 & 176 & 264 & 4,91 \\
\hline 3 & República Argentina & 42 & 177 & 219 & 4,07 \\
\hline 4 & Mariano Moreno & 7 & 178 & 185 & 3,31 \\
\hline 5 & San Miguel & 35 & 130 & 165 & 3,07 \\
\hline 6 & Don Bosco & 42 & 122 & 164 & 3,06 \\
\hline 7 & Simón Bolívar & 31 & 127 & 158 & 2,94 \\
\hline 8 & San Francisco & 17 & 126 & 143 & 2,66 \\
\hline 9 & Independencia & 5 & 133 & 138 & 2,57 \\
\hline 10 & El Porvenir & 40 & 90 & 130 & 2,42 \\
\hline 11 & San Antonio & 28 & 99 & 127 & 2,36 \\
\hline 12 & Villa Lourdes & 14 & 108 & 122 & 2,27 \\
\hline 13 & Lisbel A. Rivira & 36 & 83 & 119 & 2,21 \\
\hline 14 & Nueva Formosa & 22 & 88 & 110 & 2,04 \\
\hline
\end{tabular}




\begin{tabular}{llcccc}
\hline Orden & Barrios & $\begin{array}{c}\text { Delitos } \\
\text { contra las } \\
\text { personas }\end{array}$ & $\begin{array}{c}\text { Delitos } \\
\text { contra la } \\
\text { propiedad }\end{array}$ & $\begin{array}{c}\text { Total de } \\
\text { delitos }\end{array}$ & $\begin{array}{c}\text { \% de } \\
\text { delitos }\end{array}$ \\
\hline 15 & La Floresta & 21 & 80 & 101 & 1,88 \\
\hline 16 & 2 de Abril & 14 & 86 & 100 & 1,87 \\
\hline 17 & Antenor Gauna & 21 & 70 & 91 & 1,69 \\
\hline 18 & Villa Del Rosario & 8 & 80 & 88 & 1,64 \\
\hline 19 & 7 de Mayo & 33 & 54 & 87 & 1,63 \\
\hline 20 & Obrero & 17 & 70 & 87 & 1,63 \\
\hline 21 & Virgen de Guadalupe & 18 & 67 & 85 & 1,58 \\
\hline 22 & Juan Domingo Perón & 30 & 54 & 84 & 1,56 \\
\hline 23 & 20 de Julio & 18 & 63 & 81 & 1,51 \\
\hline 24 & Vial & 7 & 74 & 81 & 1,51 \\
\hline 25 & La Paz & 7 & 72 & 79 & 1,47 \\
\hline
\end{tabular}

Fuente: elaboración propia con datos del Comando Radioeléctrico Policía de la Provincia de Formosa.

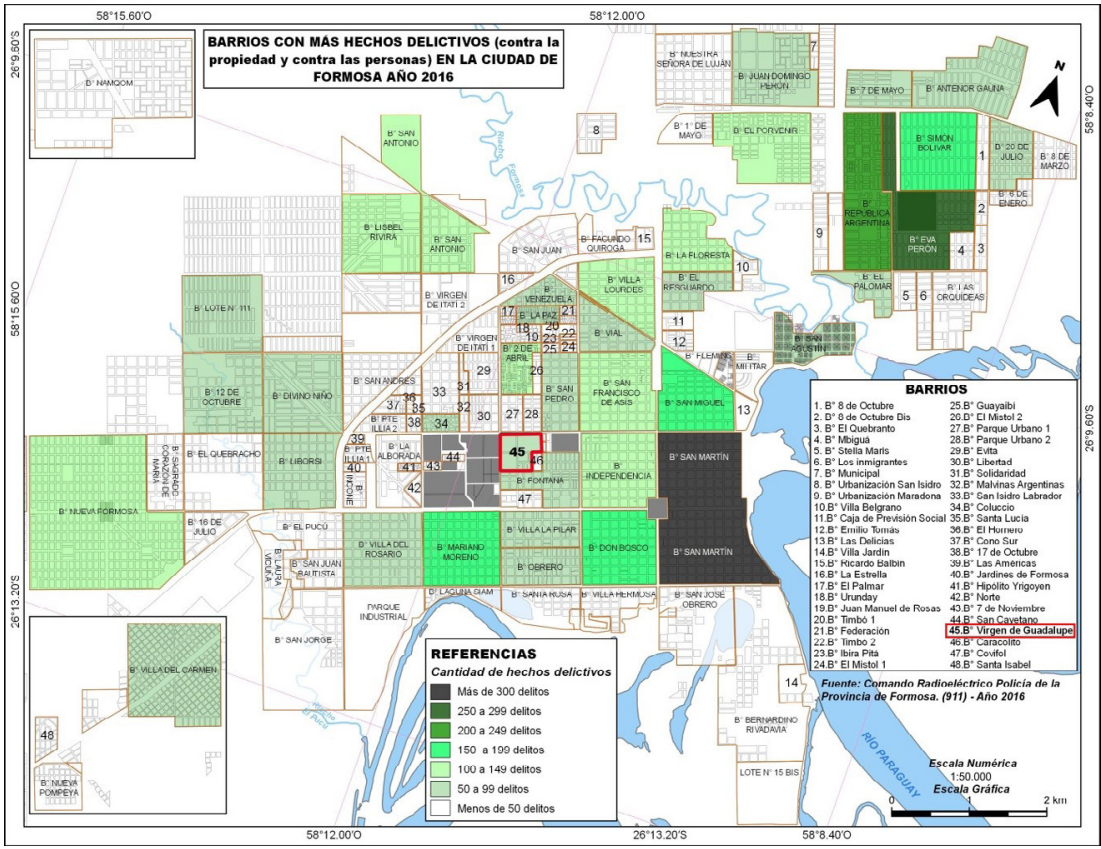

Figura 7. Barrios con más hechos delictivos en la ciudad de Formosa, 2016. Fuente: elaboración propia con datos del Comando Radioeléctrico de la Policía de la Provincia de Formosa. 
En el caso que nos compete, el barrio Virgen de Guadalupe, se puede ver que apenas el 1,58\% del total de delitos de toda índole ( 85 hechos) cometido durante el año 2016 en toda la ciudad de Formosa se produjeron en ese complejo habitacional, lo que habla claramente de la baja ocurrencia delictiva en el barrio.

De esos 85 hechos delictivos cometidos en el barrio, 18 de ellos fueron contra las personas y 67 contra la propiedad. Este sector residencial ocupa el vigésimo primer lugar en la grilla de hechos delictivos por barrio dentro de la ciudad capital.

En el plano de la Figura 7, se puede ver la proporción de delitos por barrio en la capital formoseña.

\section{Conclusiones}

En base al análisis realizado, se puede determinar que la cantidad de delitos ocurridos en el barrio estudiado es muy inferior si la comparamos con la percepción de inseguridad que de él poseen los ciudadanos formoseños. Se puede afirmar que el barrio virgen de Guadalupe soporta un importante grado de estigmatización residencial, en vista a los resultados obtenidos de la investigación llevada a cabo.

Esta situación se refleja en el alto porcentaje de vecinos que consideran a este barrio uno de los dos más inseguros de la capital formoseña influenciados por las opiniones vertidas por los medios de comunicación y de las redes sociales.

Consideramos una situación de estigmatización residencial cuando más de la mitad de las personas consultadas manifiestan que determinado lugar es inseguro porque han estado influenciados por la opinión vertida mediante de los medios de comunicación social y las redes sociales. En el caso de este complejo habitacional en particular, un 51,2\% de los entrevistados admite que formó su opinión y/o percepción influenciado por estos medios y redes. Esto se visualiza en el bajo número de los hechos delictivos ocurridos en este vecindario, (casi el 1,6\% de los delitos totales registrados por el Comando Radioeléctrico de la Policía de la Provincia de Formosa en el año 2016 para toda la ciudad capital) lo que no altera la imagen negativa que los vecinos poseen del barrio (más del $31,6 \%$ de los encuestados lo ven con imagen topofóbica respecto a la inseguridad).

Esto reafirma las expresiones de Bergman et al. (2008) sobre la sensación de inseguridad, la que aumenta cuando los delitos se incrementan, pero no disminuye cuando los hechos delictivos lo hacen, esta concepción es aplicable al barrio en estudio. Tal es asi, que el barrio queda sometido a un etiquetamiento social que no solo aplica a los malvivientes, sino a todos los vecinos, lo que trae aparejado sentimientos contrapuestos y frustrantes.

En definitiva, con los resultados de la investigación actual, podemos asegurar que existe un considerable grado de estigmatización residencial en 
el barrio Virgen de Guadalupe, producto de la percepción negativa que de él tienen los vecinos de la ciudad de Formosa, cuyas causas se sitúan en torno al consumo de información que ofrecen los medios masivos de comunicación y de las redes sociales, tal como lo manifestaron los ciudadanos encuestados.

Se considera que, ante la escasez o inexistencia de trabajos sobre la temática en la ciudad de Formosa, éste es un buen punto de partida para: a) articular esfuerzos entre el ámbito académico y la gestión de políticas públicas estatales, con la finalidad de brindar mejores respuestas ante un problema social; b) continuar ampliando el análisis e interpretación de una realidad cada vez más compleja de la sociedad actual.

Queda por delante desde al ámbito académico, ampliar la investigación incorporando otros aspectos al análisis de la problemática de la "percepción del delito" y la "ocurrencia del delito", como por ejemplo la indagación sobre "el lugar de residencia" de los delincuentes, es decir, la respuesta a la pregunta sobre dónde viven las personas que cometen los delitos, cuáles son los barrios donde han vivido. Un gran aporte haría la visión de otras especialidades científicas en estudios sobre el manejo, manipulación y en todo caso influencia de los medios masivos de comunicación y redes sociales en la formación de la opinión pública, como también las proporciones y tipos de delitos no denunciados y las causas de no realizar la denuncia.

\section{Referencias}

Barinas Salcedo, J. A. (2014). Aportes de la Geografía Humanística para la comprensión de los lugares de miedo al delito en la ciudad. Revista Perspectiva Geográfica, 19(2), 241-258. http://doi.org/10.19053/01233769.4093

Bergman, M., \& Kessler, G. (2008). Vulnerabilidad al delito y sentimiento de inseguridad en Buenos Aires: Determinantes y consecuencias. Desarrollo Económico. Revista de Ciencias Sociales. 190(48), 209-234. http//www.jstor.org/stable/27667837?seq=1\#page_scan_tab_contents

Capron, G. (2009). Geografías del miedo, individualismo y cambios en los modos de vida metropolitana. En Mahecha (Ed.), Globalización y territorio: reflexiones geográficas en América Latina (pp. 159-180.). Bogotá, Colombia: Universidad Nacional de Colombia.

Carman, M., Neiva V., \& Segura, R. (2013.) Introducción. Antropología, diferencia y segregación urbana. En M. Carman, V. Neiva, \& R. Segura (Coords.), Segregación y diferencia en la ciudad (pp. 11-34). Quito, Ecuador: Facultad Latinoamericana de Ciencias Sociales, Flacso, Ecuador; Consejo Latinoamericano de Ciencias Sociales (Clacso); Ministerio de Desarrollo Urbano y Vivienda.

Censo Nacional de Población y Vivienda 2010. Provincia de Formosa. INDEC. http://www.indec.gov.ar/

Conte, R. O. (2000). Formosa. Una perspectiva histórica y urbana. [Tesis de Doctorado, Universidad del Salvador], Buenos Aires. 
Conte, R. O. (2017). La percepción del delito en la ciudad de Formosa. Análisis de los diferentes sectores urbanos. En Ramírez (Coord.), Actas del VI Congreso Nacional de Geografía de Universidades Públicas- República Argentina y XVI Encuentro de Profesores en Geografía del Nordeste Resistencia-Chaco (pp. 1-14). Resistencia. Instituto de Investigaciones Geohistóricas (IIGHI)-Consejo Nacional de Investigaciones Científicas y Técnicas (CONICET)-Universidad Nacional del Nordeste (UNNE).

Conte, R. O. (Dir.) (2016). Geodimensionando al delito. La ciudad de Formosa y los territorios de la inseguridad. Proyecto de investigación SECYT-UNAF N 54/ H129. Secretaría General de Ciencia y Técnica. Universidad Nacional de Formosa.

Conte, R. O. (2016). La percepción de la inseguridad en la ciudad de Formosa: el caso del barrio Obrero. Revista Párrafos Geográficos. 15(2), 50-72. http://igeopat.org/parrafosgeograficos/index.php.

Diario Noticias Formosa. http//www.noticiasformosa.com.ar

Elorza, A. (2019). Segregación residencial y estigmatización territorial. Representaciones y prácticas de los habitantes de territorios segregados. Revista Eure. 45(135), http://doi.org/10.4067/S0250-71612019000200091

Flores Gutiérrez, S. (2015). La percepción de la inseguridad y el miedo al delito en los diagnósticos de inseguridad. Una propuesta metodológica desde la Geomática. [Tesis de Maestría, CONACYT-CENTROGEO]. https://centrogeo. repositorioinstitucional.mx/jspui/bitstream/1012/217/1/70-2016-\%20 04-2015-Tesis-Flores\%20Guti\%2B\%C2\%AErrez\%2C\%20Socorro-Maestra\%20 en $\% 20 \mathrm{Geom} \%$

Fraile, P. (2007). La percepción de seguridad: entre el delito, el conflicto y la organización del espacio. Revista Scripta Nova, 11(245), 1-10. http://www.ub.edu/geocrit/sn/sn-24562.htm

Fuentealba Carrasco, P., Rojas González, J. S., \& Barriga, O. A. (2016). Diferencias en la percepción de inseguridad a nivel cognitivo y emocional de acuerdo al perfil sociodemográfico y político. Estudio del Gran Concepción, Chile. Revista Política Criminal, 11(22), 620-655.

http://doi.org/10.4067/S0718-33992016000200008

Google Earth (2020). Imagen satelital de la ciudad de Formosa.

Google Street View (julio, 2019). Imágenes del barrio Virgen de Guadalupe.

Infraestructura de Datos Espaciales de Formosa (IDEF) (2020).

Kessler, G. (2012). Las consecuencias de la estigmatización territorial. Reflexiones a partir de un caso en particular. Revista Espacios en Blancos. Serie Indagaciones (22), 165-197. https://www.redalyc.org/revista.oa?id=3845

Kessler, G. (2009). El sentimiento de inseguridad. Sociología del temor al delito. Editorial Siglo XXI, Buenos Aires.

Lindón, A. (2007). La ciudad y la vida urbana a través de los imaginarios urbanos. Revista Eure. 99(33), 7-16. http://www.scielo.cl/pdf/eure/v33n99/art02.pdf

Martínez, M. (2020). Percepción y ocurrencia del delito en el barrio Virgen de Guadalupe de la ciudad de Formosa entre 2014 y 2016. ¿Inseguridad o estigmatización 
residencial? [Tesina de licenciatura, Universidad Nacional de Formosa] Formosa.

Mape Guzmán, F. \& Avendaño Arias, J. (2017). Topofobias e imaginarios del miedo sobre el espacio urbano de la localidad de Fontibón, Bogotá, Colombia. Perspectiva Geográfica, 22(1). http://doi.org/10.19053/01233769.6115

Moreno, C. (2012). Vulnerabilidad al delito y sentimiento de inseguridad en las grandes áreas urbanas de la Argentina: factores que influyen en el riesgo de victimización y disparan el miedo al delito. Boletín de Inseguridad. Observatorio de la deuda social argentina. Pontificia Universidad Católica Argentina, 1-12. http://www.uca.edu.ar/uca/common/grupo81/files/boletin

Policía de la Provincia de Formosa. Dirección de Informaciones Policiales, Comando Radioeléctrico 911. Datos de ocurrencia del delito por barrios, año 2016.

Pyszczek, O. L. (2012). Los espacios subjetivos del miedo: construcción de la estigmatización espacial en relación con la inseguridad delictiva urbana. Revista Cuadernos de Geografía. 1(21) 41-54. http://www.revistas.unal.edu.co/index.php/rcg/article/view/30694/36757

Segura, R. (2012). Elementos para una crítica de la noción de segregación residencial socioeconómica: desigualdades, desplazamientos e interacciones en la periferia de La Plata, Revista Quid, 16(2), 106-132. https://bit.ly/2TunSNn

Segura, R. (2009). Paisajes del miedo en la ciudad. Miedo y ciudadanía en el espacio urbano de la ciudad de La Plata. Revista Cuaderno Urbano. Espacio, Cultura y Sociedad, 8(8), 59-91.

http//arq.unne.edu.ar/publicaciones/cuaderno-_urbano/cu_8/index.html

Segura, R. (2006). Segregación residencial, fronteras urbanas y movilidad territorial. Un acercamiento etnográfico. Cuadernos del IDES 9. https://publicaciones.ides. org.ar/sites/default/files/docs/2020/cuadernosdelides-9-2006-segura.pdf

Tecco, C. A. y Fernández, S. C. (2008) Espacios urbanos estigmatizados, segregación residencial y agenda pública local. Revista Administración Pública y Sociedad (16), 1-18. http://biblioteca.municipios.unq.edu.ar/modules/mislibros/ archivos/Tecco-Fern\%E1ndez\%20AP\&S\%20N\%BA\%2016.pdf

Urteaga, E. (2012) Segregación y estigmatización de los barrios desfavorecidos en Francia. Revista Portularia, 12(1), 39-47. https://doi.org/10.5218/prts.2012.0032

Van Soomeren, P. (2007). El delito y la inseguridad subjetiva desde la arquitectura y el urbanismo. Revista DSP-Groep, (34), 241-287. https://publicaties.dsp-groep. nl/getFile.cfm?file=18pvciudades_artikel.pdf\&dir=rapport

Vera, A., Ávila, M., Martínez-Ferrer, B., Musitu, G. \& Montero, D. (2017). Percepción de inseguridad, victimización y restricciones en la vida cotidiana en función del ciclo vital, en Morelos, México. Revista Criminalidad, 59(3), 183-192. https://www.researchgate.net/publication/322065063_Percepcion_de_ inseguridad_victimizacion_y_restricciones_en_la_vida_cotidiana_en_ funcion_del_ciclo_vital_en_Morelos_Mexico

Wacquant, L. Slater, T., \& Borges Pereira, V. (2014). Estigmatización territorial en acción. Revista INVI. 29(82), 219-240.

https://dpoi.org/10.4067/S0718-83582014000300008 